\title{
Identifying Specific Light Inputs for Each Subgroup of Brain Clock Neurons in Drosophila Larvae
}

\author{
André Klarsfeld, ${ }^{1,2}$ Marie Picot, ${ }^{1,2}$ Carine Vias, ${ }^{1,2}$ Elisabeth Chélot, ${ }^{1,2}$ and François Rouyer ${ }^{1,2}$ \\ ${ }^{1}$ Institut de Neurobiologie Alfred Fessard, CNRS UPR 3294, Centre National de la Recherche Scientifique, 91198 Gif-sur-Yvette, France, and ${ }^{2}$ Département \\ de Biologie, Université Paris Sud, 91405 Orsay, France
}

In Drosophila, opsin visual photopigments as well as blue-light-sensitive cryptochrome (CRY) contribute to the synchronization of circadian clocks. We focused on the relatively simple larval brain, with nine clock neurons per hemisphere: five lateral neurons (LNs), four of which express the pigment-dispersing factor (PDF) neuropeptide, and two pairs of dorsal neurons (DN1s and DN2s). CRY is present only in the PDF-expressing LNs and the DN1s. The larval visual organ expresses only two rhodopsins (RH5 and RH6) and projects onto the LNs. We recently showed that PDF signaling is required for light to synchronize the CRY ${ }^{-}$larval DN2s. We now show that, in the absence of functional CRY, synchronization of the DN1s also requires PDF, suggesting that these neurons have no direct connection with the visual system. In contrast, the fifth $\left(\mathrm{PDF}^{-}\right) \mathrm{LN}$ does not require the PDF-expressing cells to receive visual system inputs. All clock neurons are light-entrained by light-dark cycles in the $r h 5^{2} ; c r y^{b}, r h 6^{1} c r y^{b}$, and $r h 5^{2} ; r h 6^{1}$ double mutants, whereas the triple mutant is circadianly blind. Thus, any one of the three photosensitive molecules is sufficient, and there is no other light input for the larval clock. Finally, we show that constant activation of the visual system can suppress molecular oscillations in the four PDF-expressing LNs, whereas, in the adult, this effect of constant light requires CRY. A surprising diversity and specificity of light input combinations thus exists even for this simple clock network.

\section{Introduction}

Endogenous clocks control many self-sustained rhythms in physiology and behavior with $\sim 24$ h periodicity (Dunlap et al., 2004). These clocks are thus called circadian (from Latin "approximately a day"), and need daily synchronization to the outside world, achieved mainly via specific light inputs (Roenneberg et al., 2003). In Drosophila, investigations of such synchronization (or entrainment) have focused mainly on the $\sim 80$ adult clock neurons located in each brain hemisphere (Nitabach and Taghert, 2008). These neurons are divided into several lateral and dorsal groups (Helfrich-Förster et al., 2007b). Light acts both through

Received Oct. 1, 2010; revised Sept. 14, 2011; accepted 0ct. 3, 2011.

Author contributions: A.K., M.P., and F.R. designed research; A.K., M.P., C.V., and E.C. performed research; A.K., M.P., E.C., and F.R. analyzed data; A.K. and F.R. wrote the paper.

This work was supported by Action Concertée Incitative "Biologie du développement et physiologie intégrative" from Ministère de l'Enseignment Supérieur et de la Recherche (MESR), the Agence Nationale de la Recherche DrosoClock and ClockNet projects, and European Union sixth framework Entrainment of the Circadian Clock (EU(LOCK) project to F.R. M.P. was supported successively by MESR and Fondation pour la Recherche Médicale and F.R. by the Institut National de la Santé et de la Recherche Médicale. We thank B. Richier and M. Boudinot for their expertise with the imaging system, L. Collet for artwork, and T. Guignard and C. Michard-Vanhée for helpful discussions. L. Gouny contributed some preliminary experiments during her master's internship. We thank the IMAGIF facility for confocal microscopy. We are grateful to J. Champagnat for his strong support. We also thank S. Britt, C. Desplan, P. Hardin, M. Rosbash, R. D. Shortridge, and R. Stanewsky for antibodies and/or fly strains, and S. Sprecher for communicating results before publication.

Correspondence should be addressed to either André Klarsfeld or François Rouyer, Institut de Neurobiologie Alfred Fessard, CNRS UPR 3294, 1 av. de la Terrasse, 91198 Gif-sur-Yvette Cedex, France, E-mail: rouyer@inaf. cnrs-gif.fr or andre.klarsfeld@espci.fr.

A. Klarsfeld's present address: Laboratoire de Neurobiologie, CNRS UMR 7637, Ecole Supérieure de Physique et de Chimie Industrielles ParisTech, 75005 Paris, France.

M. Picot's present address: CNRS UMR 7224 and INSERM UMR 952, Université Pierre-et-Marie Curie, 75252 Paris Cedex 05, France.

DOI:10.1523/JNEUROSCI.5159-10.2011

Copyright $\odot 2011$ the authors $\quad 0270-6474 / 11 / 3117406-10 \$ 15.00 / 0$ rhodopsin-containing photoreceptors and the blue-lightsensitive protein CRYPTOCHROME (CRY), which is expressed in many but not all clock neurons (Benito et al., 2008; Yoshii et al., 2008). Retinal, ocellar, and extraretinal "eyelet" photoreceptors (Hofbauer and Buchner, 1989) are all involved (Rieger et al., 2003; Veleri et al., 2007). At least five rhodopsins (including RH1, RH5, and RH6) contribute to clock entrainment (Hanai et al., 2008; Hanai and Ishida, 2009). Some clock neurons, especially the ventral lateral neurons (LNvs), may be direct targets of visual fibers (Helfrich-Förster et al., 2002, 2007a; Malpel et al., 2002; Yasuyama et al., 2006).

LNvs express the neuropeptide pigment-dispersing factor (PDF) (Helfrich-Förster, 1995), which differentially affects entrainment and rhythmicity of many clock neurons, including the PDF $^{-}$fifth s-LNv (Peng et al., 2003; Klarsfeld et al., 2004; Lin et al., 2004; Wülbeck et al., 2008; Cusumano et al., 2009; Yoshii et al., 2009).

The larva brain clock network is much simpler than that of the adult (Kaneko et al., 1997) and is made up of five LNs, four of which express PDF, and two pairs of dorsal neurons (DNs), the DN1s and the DN2s. They all survive into the adult stage (Kaneko and Hall, 2000; Shafer et al., 2006). Only the $\mathrm{PDF}^{+}$LNs and the DN1s express CRY (Klarsfeld et al., 2004). The larval visual system, the Bolwig organ (BO), comprises only 12 cells. They express either RH5 or RH6, which are the two rhodopsins used by adult R8 photoreceptors (Malpel et al., 2002; Sprecher et al., 2007). The eight RH6-expressing cells die during metamorphosis, while the other four switch to RH6 expression (Sprecher and Desplan, 2008), and form the eyelet (Helfrich-Förster et al., 2002; Sprecher and Desplan, 2008). Both types of larval photoreceptors 

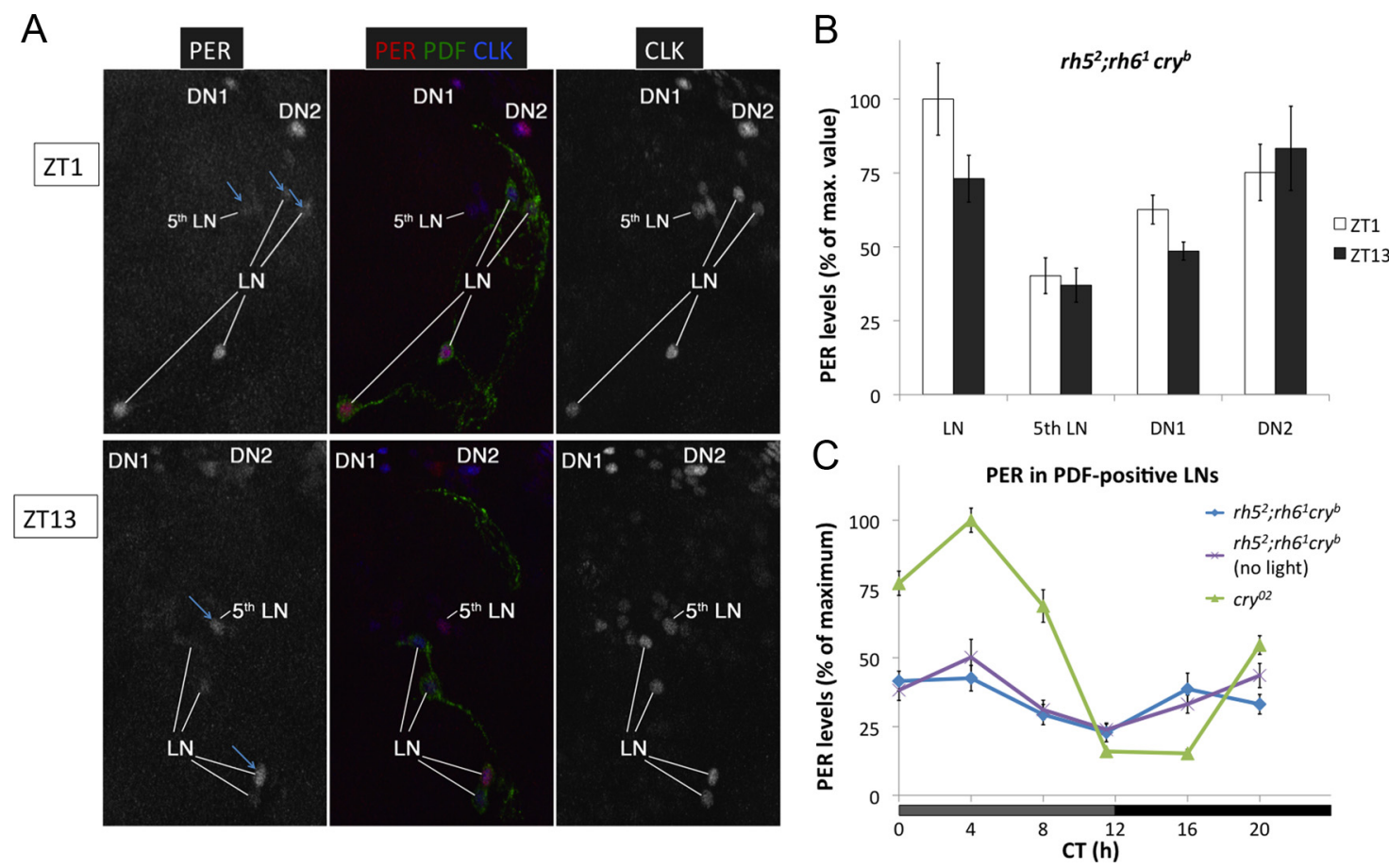

Figure 1. PER oscillations in larval brains of triply defective photoreceptive mutants entrained in $L D$. Larvae were entrained in $L D$ conditions for $5 \mathrm{~d}\left(25^{\circ} \mathrm{C}\right)$ before dissections. Similar results were obtained in at least two experiments for each genotype. A, PER immunoreactivity (left, and red in middle) in $r h 5^{2} ;$; $h 6^{1} \mathrm{cry}^{b}$ mutant brains at ZT1 (light-on) and ZT13 (1 h after light-off), from projections of Apotome confocal stacks. PDF labeling (green in middle) was used to distinguish the four PDF ${ }^{+}$LNs, and GP47 labeling (right, and blue in middle) was used to confirm localization of the other five clock neurons, as CLOCK-expressing cells. Arrows point to LNs that are very weakly labeled at ZT1 (when PER is strongly expressed in wild-type larvae) or strongly labeled at ZT13 (when PER expression is very low in wild-type larvae). There was no significant correlation between mean PER levels in the LNs in the left and right hemispheres of a given brain ( $r=0.21$ and $p=0.56$, when pooling ZT1 and ZT13 values). Weak PER expression was also detected in a few additional GP47-labeled cells, most likely adult-specific clock neurons, which do not display molecular oscillations until mid-metamorphosis (Kaneko and Hall, 2000; Helfrich-Förster et al., 2007). B, PER oscillations in different groups of larval clock neurons of $r h 5^{2}$; rh $6^{1} c r y^{b}$ mutants. PER labeling was quantified separately in the four PDF ${ }^{+} \mathrm{LNs}$, the fifth LN, the DN1s, and the DN2s, as indicated. Values were averaged over at least 12 brain hemispheres. They are expressed as percentage of the maximal average value. In this particular experiment, the maximal average value was the average of the LNs at ZT1. Error bars represent the SEM for each neuronal group. C, PER oscillations in the $\mathrm{PDF}^{+} \mathrm{LNs}$ of $r h 5^{2} ; r h 6^{1} \mathrm{cry}{ }^{b}$ mutant larvae, with or without prior exposure to $\mathrm{LD}$ cycles. Dissections were performed every $4 \mathrm{~h}$ on the first day in DD. Labeling is expressed as percentage of the peak in cry ${ }^{02}$ control brains, which were dissected on the second day in DD. The highest PER levels in LNs of triple mutant brains, when pooling all time points (data not shown), were similar to the cry ${ }^{02}$ peak. Relative standard deviations of PER labeling were, however, significantly higher in the triple mutants, in either condition, compared to control (Student's $t$ tests on the six time-point values, $p<0.01)$. Relative standard deviations were not significantly different between the light-exposed and light-shielded triple mutants $(p>0.18)$. Dark gray and black horizontal bars indicate the light and dark portions, respectively, of the preceding LD cycle.

project onto the PDF-expressing LNs (Kaneko et al., 1997; Malpel et al., 2002; Mazzoni et al., 2005). CRY and the BO provide the only light inputs into the larval clock (Kaneko et al., 2000; Malpel et al., 2004). Synchronization of the larval CRY ${ }^{-}$DN2s, which display PERIOD protein (PER) oscillations almost in antiphase to all other clock neurons (Kaneko et al., 1997), requires PDF signaling from the LNs (Picot et al., 2009).

Here we show that PDF signaling can also synchronize the larval DN1s, whereas it does not participate in the synchronization of the fifth $\mathrm{PDF}^{-} \mathrm{LN}$. We also show that both larval rhodopsins are involved in clock responses to light. Together with CRY, they provide the only light inputs available to the larval clock. Unexpectedly, constant stimulation of the visual system disrupts PER oscillations specifically in the PDF-expressing larval LNs and independently of CRY. Larval exposure to constant red light indeed perturbs the clock much more strongly than anticipated from experiments in the adult.

\section{Materials and Methods}

Strains. Drosophila cultures were usually maintained on a $12 / 12 \mathrm{~h} \mathrm{light/}$ dark (LD) cycle on standard corn meal-yeast-agar medium at $25^{\circ} \mathrm{C}$ and $50 \%$ relative humidity. Control strains were laboratory stocks of $w$ or $y w$ flies, which behaved similarly. Researchers have described $p d f^{01}$ (Renn et al., 1999), $c r y^{b}$ (Stanewsky et al., 1998), $c r y^{02}$ (Dolezelova et al., 2007), UAS-cry (Emery et al., 2000b), cry-gal4-39 (Klarsfeld et al., 2004),
w;;Gal1118 (Blanchardon et al., 2001), cry-gal80 (Stoleru et al., 2004), GMR-hid (Bergmann et al., 1998), and yw UAS-hid UAS-rpr (Zhou et al., 1997) lines. The $r h 6^{1}$ mutation is a $20 \mathrm{bp}$ spontaneous deletion present in some laboratory stocks, resulting in a null allele (Misra et al., 2002). The $r h 5^{2}$ mutation is a complete ORF deletion (Yamaguchi et al., 2008). All combinations of $r h 5^{2}, r h 6^{1}, p d f^{01}$, and $c r y^{b}$ flies, as well as GMR-hid;cry ${ }^{02}$ flies, were produced by standard crosses and recombinations. For the mutant genotypes tested in constant light (LL), as well as for the controls in constant red light (RR), all results were similar when using strains with the $l s$-tim allele [encoding a TIMELESS protein (TIM) isoform that is less sensitive to CRY-dependent light-induced degradation] (Peschel et al., 2006). All clock neurons of the larval brain and all clock neurons of the adult brain express $y$ w;tim-gal4. Several other neuronal groups that do not express clock genes also express yw;tim-gal4 (Kaneko, 1998). The $U A S-G F P$ and UAS-CD8-GFP reporter lines, which were provided by the Bloomington stock center, carry their insertion on the second chromosome.

Culture and entrainment conditions. LD-grown flies were crossed and placed in $\mathrm{LD}$ conditions at $25^{\circ} \mathrm{C}$. At this temperature, the offspring reach third-instar wandering stage in $\sim 5 \mathrm{~d}$. Cultures were thus entrained in LD 12:12 h for 5 or $6 \mathrm{~d}$ before larvae were dissected (for LD time points), or entrained in LD 12:12 h for $4 \mathrm{~d}$ and transferred into constant darkness (DD) (for DD time points), or into constant light at least $24 \mathrm{~h}$ before first dissection time (considered then as CT24). One experiment included both entrained and unentrained $r h 5^{2} ; r h 6^{1} c r y^{b}$ larvae. The latter were kept in the same incubators, with aluminum foil wrapped around the tubes so 
the larvae never saw light. For constant red light, the culture tubes were put into opaque tin cans covered with a red filter (cutoff: $600 \mathrm{~nm}$ ) after the last LD cycle (Cusumano et al., 2009). Simultaneous Gal1118-driven expression of the proapoptotic genes hid and rpr eliminates the LNs by the third larval stage under standard culture conditions (Malpel et al., 2002). For the present LN ablation experiments, larvae were first kept in constant darkness for 3-4 d, and then entrained for $3 \mathrm{~d}$. We checked that brain PDF projections were already absent before such larvae were exposed to their first entraining LD cycle $(n=14$ hemispheres stained with anti-PDF antiserum). In contrast, $\mathrm{PDF}^{+}$cells at the tip of the abdominal ganglion were unaffected (data not shown). Temperature entrainment was performed as described previously (Picot et al., 2009).

Histology and imaging. All experiments, performed at least twice with similar results, were done on whole-mounted third-instar larval brains, which were dissected and labeled as previously described (Malpel et al., 2002). Antibodies were as previously described (Malpel et al., 2002; Picot et al., 2009), except for the use of the GP47 guinea pig anti-CLOCK (Houl et al., 2006), at 1:5000 dilution, or a guinea-pig anti-PDP1 used at 1:10,000 dilution (Benito et al., 2007). Although GP47 cross-reacts with DACHSHUND (Houl et al., 2008), the clock neurons were on average more strongly stained, and could also be distinguished based on size, shape, and location (see Figs. $1 A, 3$ ). In some experiments (e.g., that of Fig. 9A,B), tim-gal4-driven GFP expression was used to better identify clock neurons. Secondary antibodies were Alexa350-, Alexa488-, Alexa594- (or FP546- or FP568-), and FP647-conjugated goat antibodies directed against IgGs of the appropriate species (Invitrogen). They were used at 1:10,000-to-1:1000 dilutions. Images were made from epifluorescence microscopes (Zeiss) equipped with a cooled digital camera (Axiocam MR, Zeiss), and an Apotome module to generate confocal images (except for the histograms of Figs. 7, 9). Fluorescence intensity was quantified with Adobe Photoshop or ImageJ software from individual sections of at least 12 brain hemispheres per sample. We applied the formula $I=100(S-B) / B$, which gives the fluorescence percentage above background (where $S$ is fluorescence intensity and $B$ is average intensity of the region adjacent to the quantified cell). Absolute values of $I$ varied among experiments. To emphasize relative variations, values are expressed as a percentage of the maximum intensity within a given graph. In some cases, fluorescence was assessed by eye on a 0 -to- 3 scale. The two methods yielded very similar curves when performed on the same samples. Here it was used only for Figure 2, $A$ and $B$. Statistical analyses were performed with Excel. We used differences in the size of standard deviations (as percentage of means) as an index of intragroup differences in staining levels. Relative standard deviations are expected to be higher if cells oscillate with random phases than if they all express similar, constitutive PER levels (Kaneko et al., 2000; Zhang et al., 2010). Confocal imaging at higher magnification was performed on a Leica SP2 microscope for greater anatomical precision (Figs. $4 A, 5$ ). Maximum intensity projections were obtained from stacks of 5-20 confocal sections.

\section{Results}

The brain clock of $r h 5 ; r h 6^{1} c r y^{b}$ triple mutant larvae is blind Because larvae lacking CRY and the $\mathrm{BO}$ appear circadianly blind (Malpel et al., 2004), and because the BO expresses only two rhodopsins, RH5 and RH6 (Malpel et al., 2002; Sprecher et al., 2007), we tested whether removing them and CRY would prevent light entrainment of the larval brain clock. In triple mutant larvae, PER expression in all five LNs and in the DN2s was clearly unsynchronized to the LD cycle (Fig. $1 A, B$ ). PER expression varied widely from cell to cell, even within a single brain hemisphere (Fig. $1 A$ ). In contrast, PER levels in the DN1s were moderate and very similar in all such cells, at both ZT1 and ZT13. This resulted in a narrower dispersion of measurements (compare error bars for the DN1s and the DN2s in Fig. $1 B$; see also below), suggesting lack of oscillations in the DN1s versus unsynchronized oscillations in the LNs and DN2s. Similar results were obtained with GMR-hid;; $r y^{02}$ larvae (data not shown). Oscillations in LNs and $\mathrm{DN} 2 \mathrm{~s}$, in turn, suggest the molecular clock is functional in these
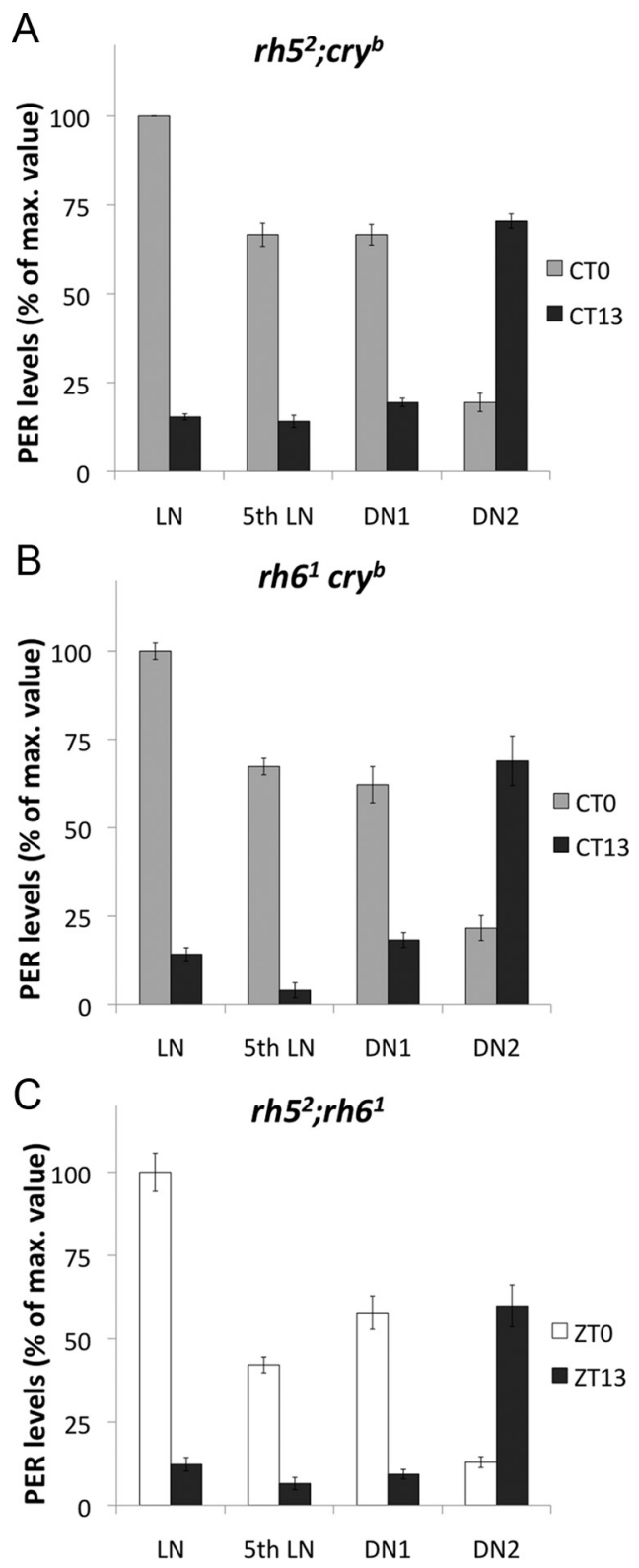

Figure 2. PER oscillations in larval brains of doubly defective photoreceptive mutants. The $r h 5^{2} ; c r y^{b}$ and $r h 6^{1} c r y^{b}$ mutants were tested on the first day in DD after LD entrainment at CTO (subjective light-on) and CT 13 (1 h after subjective light-off). Representative brains for these two genotypes are shown in Figure $3 . r h 5^{2}$; rh $6^{7}$ mutants were tested in LD at ZT0 and ZT13. Note that PER oscillations in the DN2s are in antiphase with oscillations in the other clock neurons, as previously described (Kaneko et al., 1997).

cells, but cannot be light-entrained. By contrast, temperature cycles effectively entrained all larval clock neurons (data not shown), further excluding a core clock defect in the triple mutant.

To confirm lack of entrainment of the triple mutants, we performed two additional experiments, which gave similar results, with six time points on the first day in DD. PER expression from one of these is quantified in Figure 1C, and compared to expression in larvae of the same genotype in the same experiment, but which were never exposed to light (see Material and Methods). Under both conditions, variations over time were significant, but their amplitude was much less than the cycling amplitude in control larvae. In contrast to the triple mutant, all three double mutants, with only one of the RH5, RH6, or CRY proteins still functional, displayed strong PER oscillations in all larval clock 


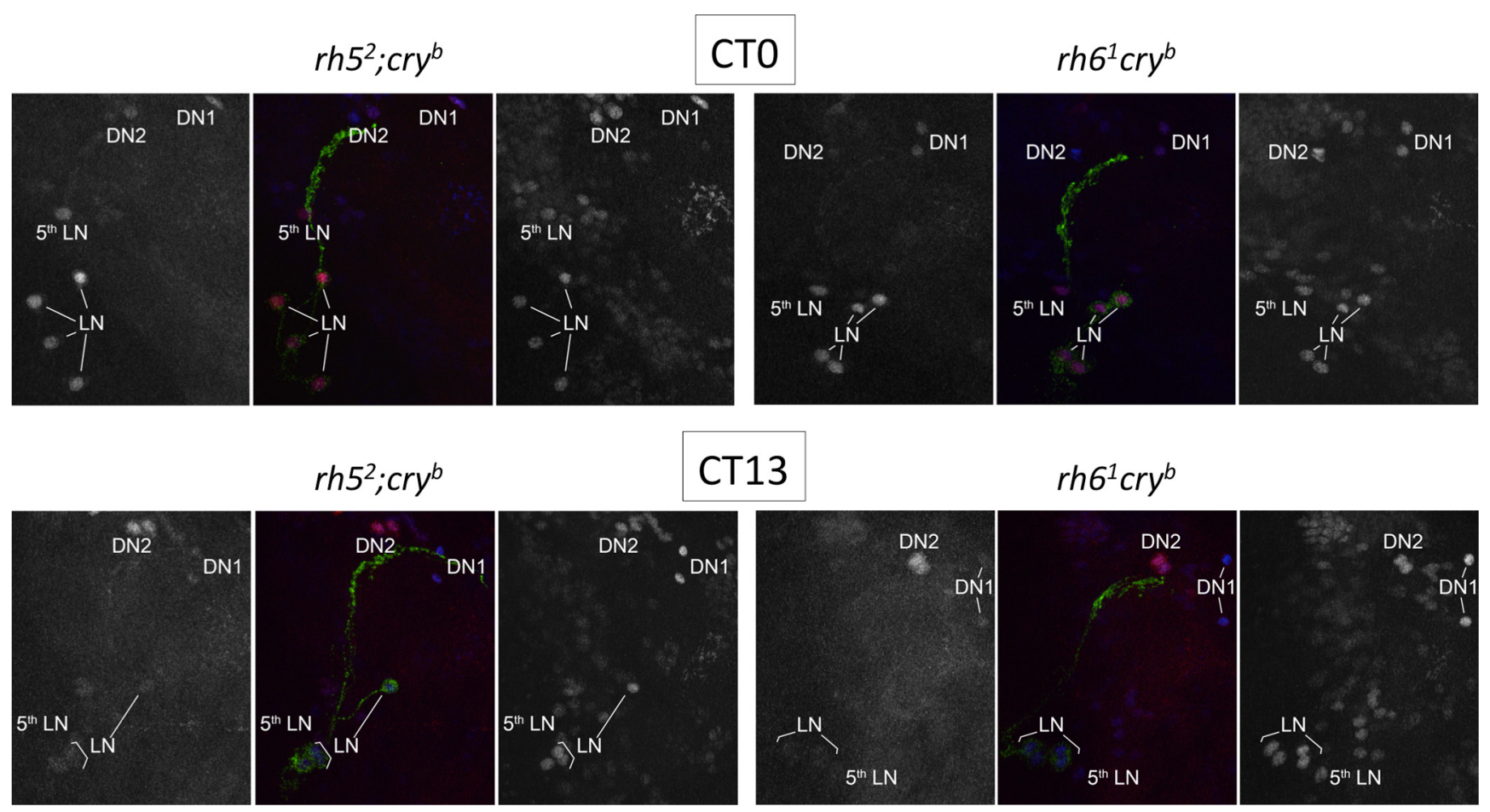

Figure 3. PER oscillations in larval brains of $r h 6^{7} c r y{ }^{b}$ and $r h 5^{2} ; c r y{ }^{b}$ mutants after LD entrainment. PER immunoreactivity is shown on the left parts of the panels (red in middle) at CTO and CT13, from projections of Apotome confocal stacks. PDF labeling (green in middle) was used to distinguish the four PDF ${ }^{+}$LNs, and anti-CLK labeling (right, and blue in middle) was used to confirm localization of the other five clock neurons.

neurons (Figs. $2 A-C, 3$ ). Together, our results thus demonstrate (1) that each of the three photoreceptive molecules is able, on its own, to entrain all larval clock neurons and (2) that together they provide the only light inputs to the larval brain clock.

\section{Entrainment of the DN1s through the visual system requires $\mathrm{PDF}$}

Because the CRY-expressing DN1s are entrained even in the $c r y^{b}$ mutant (Kaneko et al., 2000) (and Fig. 2A,B), they could be directly connected to the $\mathrm{BO}$, especially in view of their reported ventral projection toward the larval visual neuropil (Kaneko and Hall, 2000; Helfrich-Förster et al., 2007a). They could also receive an indirect input from the LNs, e.g., via PDF, like the DN2s (Picot et al., 2009). Indeed, a dendritic-like arborization from the DN1s was detected close to the dorsal projection of the LNs (Fig. 4A), similar to (but less extensive than) the arborization from the DN2s (Picot et al., 2009). When entrained by LD cycles, $c r y^{b} p d f^{01}$ larvae displayed strong PER and TIM oscillations only in the five LNs, but not in the DN2s or in the DN1s (Fig. $4 B, C$ ). The dispersion of PER and TIM labeling was significantly lower for the DN1s than for the DN2s at all time points (Fig. $4 B, C$ ), again suggesting lack of oscillations in the DN1s versus unsynchronized oscillations in the DN2s.

\section{CRY and the visual system can entrain the fifth $\left(\mathrm{PDF}^{-}\right) \mathrm{LN}$} The fifth LN appears to arborize very similarly to the $\mathrm{PDF}^{+}$LNs (Helfrich-Förster et al., 2007a), in particular at the level of larval optic neuropil (Fig. 5). The fifth LN could thus also receive direct input from the larval visual system independently of the PDF cells. This would account for its entrainment by the visual system in the absence of PDF (Picot et al., 2009). Alternatively, it could receive an indirect input through PDF-independent signaling from the PDF cells. To functionally test this possibility, we used
UAS-hid UAS-rpr; Gal1118/+ cry larvae, devoid of the $\mathrm{PDF}^{+}$ LNs (Malpel et al., 2002) and of functional CRY. PER robustly oscillated in their fifth LN, with almost no labeling at the beginning of the night (Fig. $6 A$ ). In fact, it was the only entrained clock neuron in such brains. As expected in the absence of both CRY and PDF signaling, neither of the two DN groups displayed any PER oscillations. As in Figures $1 B$ and $4, B$ and $C$, comparison of their respective error bars suggests absence of oscillations in the $\mathrm{DN} 1 \mathrm{~s}$ versus unsynchronized oscillations in the DN2s.

In the absence of a functional visual system $\left(r h 5^{2} ; r h 6^{1}\right.$ double mutant), with only CRY as photoreceptive molecule, the CRY fifth LN can still be entrained (Fig. 2C). This CRY-dependent, non-cell-autonomous entrainment pathway does not involve PDF because it is preserved in the $r h 5^{2} ; r h 6^{1} p d f^{0}$ triple mutant (Fig. 6B).

We conclude that the fifth LN can perceive light from the visual system without a relay via the $\mathrm{PDF}^{+} \mathrm{LNs}$, but rather, most likely, via a direct connection. In addition, the fifth LN can be entrained via another non-cell-autonomous pathway that is CRY dependent and PDF independent.

\section{cry mutations do not restore wild-type oscillations to the $\mathrm{PDF}^{+}$LNs in LL}

In wild-type adult flies, intense constant light abolishes activity rhythms (Emery et al., 2000a) and clamps down PER expression at very low levels in the clock neurons (e.g., Picot et al., 2007). The $c r y^{b}$ mutation restores high-amplitude behavioral and molecular rhythmicity, particularly in the s-LNvs (Emery et al., 2000a; Rieger et al., 2006; Picot et al., 2007). In the wild-type larval brain, as expected, LL abolished PER oscillations and strongly suppressed PER levels in the six CRY ${ }^{+}$, i.e., the $\mathrm{PDF}^{+} \mathrm{LNs}$ and the DN1s (Fig. 7A). The same result was obtained in GMR-hid larvae (data not shown), demonstrating that CRY is sufficient to medi- 
ate this abolition of molecular cycling. In contrast, PER oscillations in the other three clock neurons, i.e., the $\mathrm{PDF}^{-}$fifth LN and the DN2s, were little affected by LL (compare with DD on right panel of Fig. 7A). This is consistent with their complete lack of CRY expression (Klarsfeld et al., 2004). In the $c r y^{b}$ mutant, the DN1s too were not affected by LL, whereas PER oscillations and levels were still severely disrupted in the $\mathrm{PDF}^{+}$LNs (Fig. 7B). Removing either RH5 or RH6 in a $c r y^{b}$ background partially restored PER oscillations in the latter cells (Fig. 7C), suggesting that both rhodopsins contribute to producing the full effect of LL on the $\mathrm{PDF}^{+}$cells.

To confirm the suppression of oscillations, rather than a change in their phase, we performed two additional experiments in LL, with four time points. Also, to better evaluate clock function, brains were simultaneously labeled with PER, TIM, and PDP1 (and PDF) antisera. To exclude any residual CRY function that may have contributed to the $c r y^{b}$ phenotype, $c r y^{o}$ rather than $c r y^{b}$ larvae were used. Figure 8 shows quantification of PER and TIM expression in the $\mathrm{PDF}^{+} \mathrm{CRY}^{+} \mathrm{LNs}$ and in the $\mathrm{PDF}^{-} \mathrm{CRY}^{-}$fifth LN in three different genotypes. It confirms almost complete cessation of molecular rhythms specifically in the $\mathrm{PDF}^{+} \mathrm{LNs}$ of $c r y^{0}$ larvae (Fig. 8 , compare $A$ and $B$ with $C$ and $D$, respectively). PDP1 rhythms in these latter cells were also severely blunted (data not shown). In contrast, their PER and TIM oscillations were at least partly restored when one of the two larval rhodopsins was missing in a $c r y^{b}$ background (Fig. $8 A, B$ ).

\section{Constant red light abolishes wild-type PER oscillations in the $\mathrm{PDF}^{+}$larval LNs via RH6}

Because LL has a CRY-independent effect on PER oscillations in the larval $\mathrm{PDF}^{+}$ LNs, we reasoned that constant red light (>600 nm), which does not activate CRY, may disrupt PER oscillations in wild-type larvae. This was indeed the case (Fig. 9A). As expected from the results in LL with the $c r y^{b}$ mutant, PER oscillations were not affected in the other brain clock cells. The effect of RR in the $\mathrm{PDF}^{+} \mathrm{LNs}$ was strongly suppressed by the $r h 6^{1}$ (Fig. $9 B$ ) but not the $r h 5^{2}$ mutation (Fig. 9C), consistent with the described wavelength-dependent responses of the corresponding photopigments (Salcedo et al., 1999; Hanai et al., 2008). Surprisingly, RH6 and NORPA (no receptor potential A) were present all along the larval visual nerve, down to its terminal (Fig. 10). Such extended expression has not been observed in the adult visual system for either the Hofbauer-Büchner eyelet (Yasuyama and Meinertzhagen, 1999;
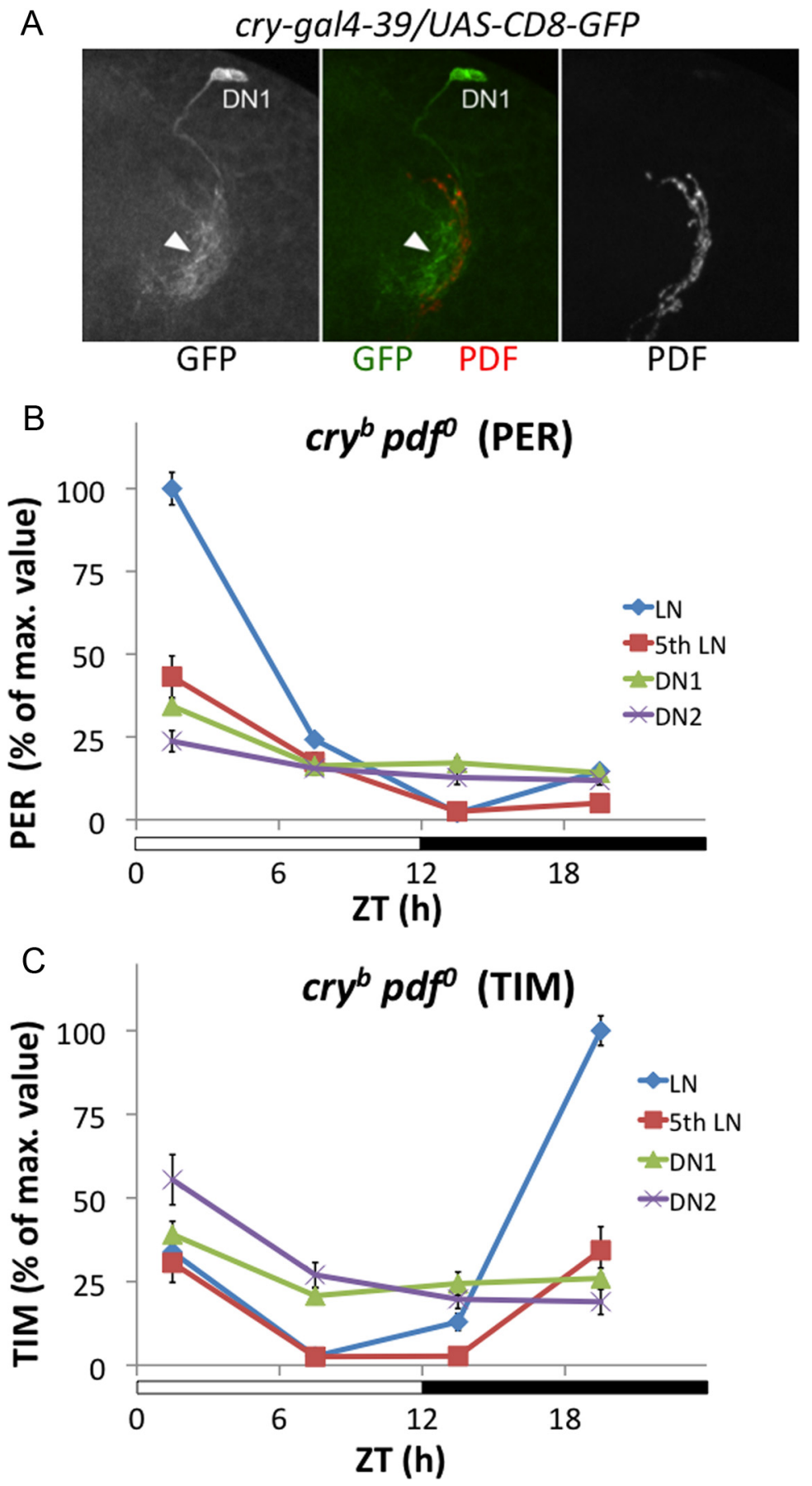

Figure 4. PDF signaling and synchronization of the DN1s. $A$, The larval DN1s have a dendritic-like arborization in close association with the PDF projections. This confocal projection was obtained from a cry-gal4 -39/UAS-CD8-GFP larval brain labeled with an anti-PDF antiserum (right, and red in middle). Note that the DN1s can be further identified from a faint cross-talk signal in the red channel, since this sample was also labeled with anti-PER. $\boldsymbol{B}, \boldsymbol{C}$, PER $(\boldsymbol{B})$ and TIM $(\boldsymbol{C})$ oscillations in $c r y^{b} p d f^{p}$ larval brains. Labeling and quantitation were performed as described in Figure 1, except that dissections were done at two additional time points in LD conditions, and that the anti-PDF antiserum was replaced by anti-TIM. Similar results were obtained in three (PER) or two (TIM) independent experiments. Relative standard deviations of PER and TIM labeling were significantly higher in the DN2s than in the DN1s (Student's $t$ tests on the four time-point values, $p<0.01$ for PER and $p<0.05$ for TIM). White and black horizontal bars indicate the light and dark portions, respectively, of the LD cycle.

Malpel et al., 2002) or the retinal photoreceptors (Wernet et al., 2006).

\section{Discussion}

The larval brain clock and its light inputs are generally considered much simpler than their adult counterparts. We find here that 

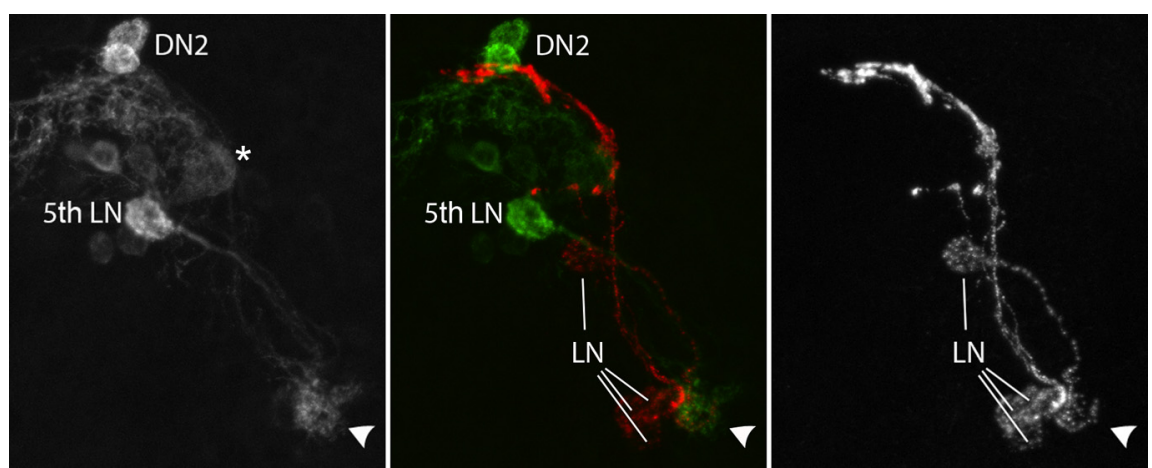

Figure 5. Arborization pattern of the fifth LN. Projection of a confocal stack from a w;tim-ga/4/UAS-CD8-GFP;cry-gal80/+ larval brain labeled with anti-PDF antibodies. Due to cry-gal80, there is no coexpression of GFP (left, and green in middle) and PDF (right, and red in middle) as can be seen clearly at the level of the PDF ${ }^{+}$somas. The fifth $\mathrm{LN}$ displays a large dendritic arborization (arrowhead) in the larval optic neuropil, where a few PDF ${ }^{+}$fibers can also be seen. Its dorsal projection is less clear, due to the extensive arborization of the DN2s, as well as weaker labeling of adult clock cells, which begin to express tim-gal 4 in the third larval stage (Helfrich-Förster et al., 2007). A large GFP ${ }^{+}$non-clock cell, which projects medially, is slightly out of focus (asterisk).
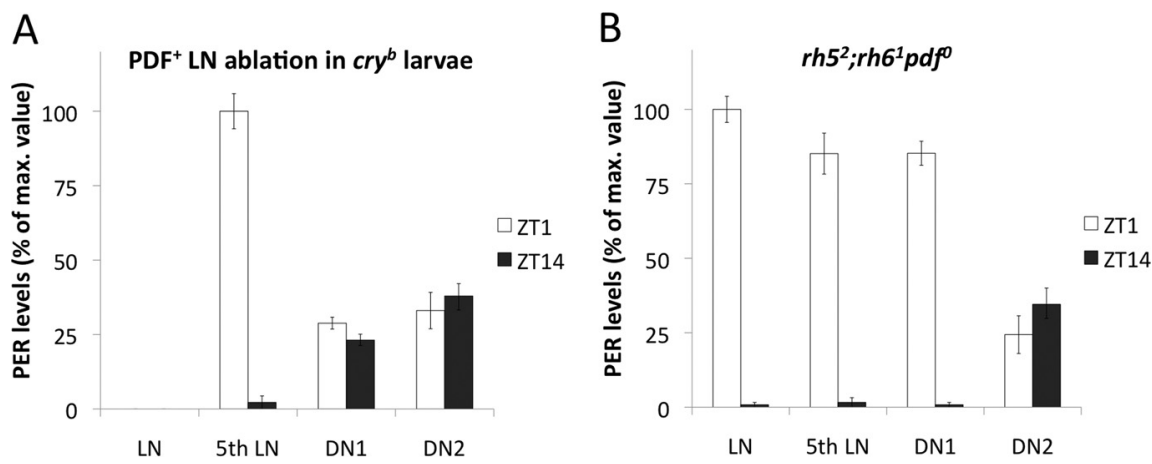

Figure 6. PER oscillations in larvae lacking either the $P D F{ }^{+} \mathrm{LNs}$ and $C R Y$, or PDF and all visual input. $\boldsymbol{A}$, Entrainment was limited to $3 \mathrm{~d}$, starting only after 3-4d in DD, to allow enough time for Gal1118-driven hid and rpr expression (initiated during the last embryonic stage) to kill the PDF ${ }^{+}$LNs before the first lights-on (see Material and Methods). Labeling and quantitation were performed as described in Figure 1 (except that only 10 brain hemispheres were used for the ZT14 time point). $\boldsymbol{B}, r h 5^{2} ; r h 6^{1} p d f^{0}$ triple mutants were entrained and dissected in parallel with the larvae used for $\boldsymbol{A}$.

larvae, with only nine clock neurons and 12 photoreceptors on each side, nevertheless display four distinct combinations of light inputs (Fig. 11 and Table 1).

Anatomical data (Kaneko et al., 1997; Malpel et al., 2002) and the present work show that $\mathrm{PDF}^{+}$LNs are the only brain cells to perceive light both cell autonomously (via CRY) and through a direct connection to the visual system. They thus appear to be the main players responsible for synchronizing the larval brain clock network to LD cycles. The DN2s, in contrast, possess neither type of light input, but play a major role in the temperature entrainment of the clock (Picot et al., 2009). We previously showed that the DN2s are intrinsically blind and must rely on PDF signaling from the LNs to synchronize to LD cycles (Picot et al., 2009). We now show that the other dorsal group, the DN1s, is also sensitive to PDF signaling. In the absence of functional CRY, PDF is required to synchronize DN1s by light, as demonstrated by the lack of PER oscillations in the DN1s of the $c r y^{b} p d f^{0}$ double mutant. This is consistent with the presence of a dendritic-like arborization from the DN1s close to the dorsal projection of the LNs. On the other hand, it tends to exclude a functional connection between the DN1s and the larval visual system, in agreement with the absence of DN1 neurites reaching the Bolwig's nerve terminals (Kaneko and Hall, 2000).

The PDF-dependent entrainment of both DN1s and DN2s by the visual system also indicates that the fifth LN, although pro- jecting largely like the $\mathrm{PDF}^{+}$LNs (Helfrich-Förster et al., 2007a), cannot synchronize the DNs. However, the fifth LN might be involved in RH5-dependent acute larval responses to light, which do not require the $\mathrm{PDF}^{+}$LNs (Hassan et al., 2005; Keene et al., 2011). The entrainment of the fifth LN in the absence of both CRY and the PDF ${ }^{+}$LNs suggests a direct connection to the visual system, in agreement with its arborization in the larval optic neuropil. Recent single-cell analysis indeed revealed this arborization to be even broader than that of the $\mathrm{PDF}^{+} \mathrm{LNs}$ (Keene et al., 2011). However, such connection to the visual system does not allow constant light to disrupt PER oscillations in the fifth LN, contrary to the $\mathrm{PDF}^{+} \mathrm{LNs}$, suggesting different downstream signaling in these two types of visual system targets. Finally, our results suggest a hitherto unsuspected connection between some $\mathrm{CRY}^{+}$ neurons and the fifth LN. This connection does not rely on PDF and could be directly from the DN1s or the $\mathrm{PDF}^{+} \mathrm{LNs}$, which both have projections in the vicinity of the fifth $\mathrm{LN}^{\prime}$ s projections.

More generally, the fact that the CRY fifth LN and DN2s display normal PER oscillations in the absence of a functional visual system is consistent with CRY transmitting light information in a noncell-autonomous way. This has already been proposed in the adult brain for the three $\mathrm{CRY}^{-}$dorsal lateral neurons and the DN2s (Helfrich-Förster et al., 2001; Yoshii et al., 2008; Cusumano et al., 2009). However, it remains possible that such nominally $\mathrm{CRY}^{-}$cells in the adult express very low levels of CRY, as judged from reporter gene expression (Klarsfeld et al., 2004). In contrast, CRY expression in the larval 5th LN and DN2s was observed neither with antibodies, not with any reporter lines. The present results make it even less likely, because constant light does not affect these neurons at all.

The role of the PDF neuropeptide in the light entrainment of the DN1s and DN2s appears somewhat different for the two subgroups. First, PDF sets the DN1s and DN2s to very different phases: the DN2s are set in antiphase with the LNs (Kaneko et al., 1997; Picot et al., 2009), whereas the DN1s are set in phase with the LNs. This suggests that the corresponding signaling cascades differ somewhere downstream from the PDF receptor. In addition, the dispersion of cell labeling intensities suggests that unentrained DN2s oscillate, although asynchronously (even within a single brain hemisphere), while unentrained DN1s do not, but rather express constant, moderate PER and TIM levels. The same may hold true for completely blind larvae $\left(r h 5^{2} ; r h 6^{1} c r y^{b}\right)$. While the LNs and DN2s seem to oscillate with random individual phases, all DN1s display very similar PER levels. This implies that, in $\mathrm{LD}, \mathrm{PDF}$ may be needed not only to synchronize but to trigger (or at least maintain until the third larval stage) DN1 oscillations in the absence of CRY activation. In contrast, PDF synchronizes persistent autonomous oscillations in the DN2s. The non-autonomous cycling of the CRY-expressing DN1s suggests that they may have an 
important role in synchronizing the network to LD cycles. Conversely, the capacity of the DN2s for autonomous cycling in the absence of light cues may relate to their specific role in temperature entrainment.

Lack of entrainment by light was previously reported for the LNs and the DN1s in norp $A^{P 41} ;$; $c r y^{b}$ larvae, while, rather surprisingly, molecular oscillations were still detected in their DN2s (Kaneko et al., 2000). While the DN2s require PDF to entrain in LD, they appear to entrain to temperature cycles very efficiently on their own (Picot et al, 2009). This means one cannot exclude the possibility that, in the Kaneko et al. (2000) study, small temperature changes induced by the LD cycles weakly entrained these neurons, but not the others in the Kaneko et al. (2000) study. Alternatively, the DN2s might collect light information from a NORPAindependent pathway. NORPA-independent photoreception appears to participate in adult circadian photoreception (Stanewsky et al., 1998).

Our results show that RH5, RH6, and CRY are the only light input pathways for synchronizing the larval clock neurons to LD cycles. RH5, RH6, and CRY are each sufficient alone to entrain all these neurons, whereas, in the adult, some clock neurons fail to entrain in the absence of CRY (Helfrich-Förster et al., 2001; Cusumano et al., 2009). At least two more rhodopsins, including RH1 and a UVblue one (RH3 and/or RH4), participate in the adult (Hanai and Ishida, 2009), so that all available rhodopsins in the adult eye may also be involved in entraining the clock. Recently, at least two classes of larval sensory neurons, outside $\mathrm{BO}$, have been shown to express visual transduction components (Shen et al., 2011; Xiang et al., 2010). One of these two is involved in thermal preferences, with $\mathrm{RH} 1$ as the presumed temperature sensor, while the other mediates rhodopsin-independent avoidance of very high light intensities. At least in the conditions used here, these novel sensory pathways do not seem to contribute to circadian light entrainment.

Interestingly, constant light, acting CRY independently through the visual system, can abolish or greatly disturb oscillations in the $\mathrm{PDF}^{+}$LNs of larvae (present results) but not adults (Peschel et al., 2006; Rieger et al., 2006; Picot et al., 2007). Similarly, the larval visual system is required for fast TIM degradation in the LNs at the end of the night (Mazzoni et al., 2005), although CRY may also be necessary (Ivanchenko et al., 2001). The $\mathrm{PDF}^{+} \mathrm{LNs}$ of eyeless adult flies, in con-
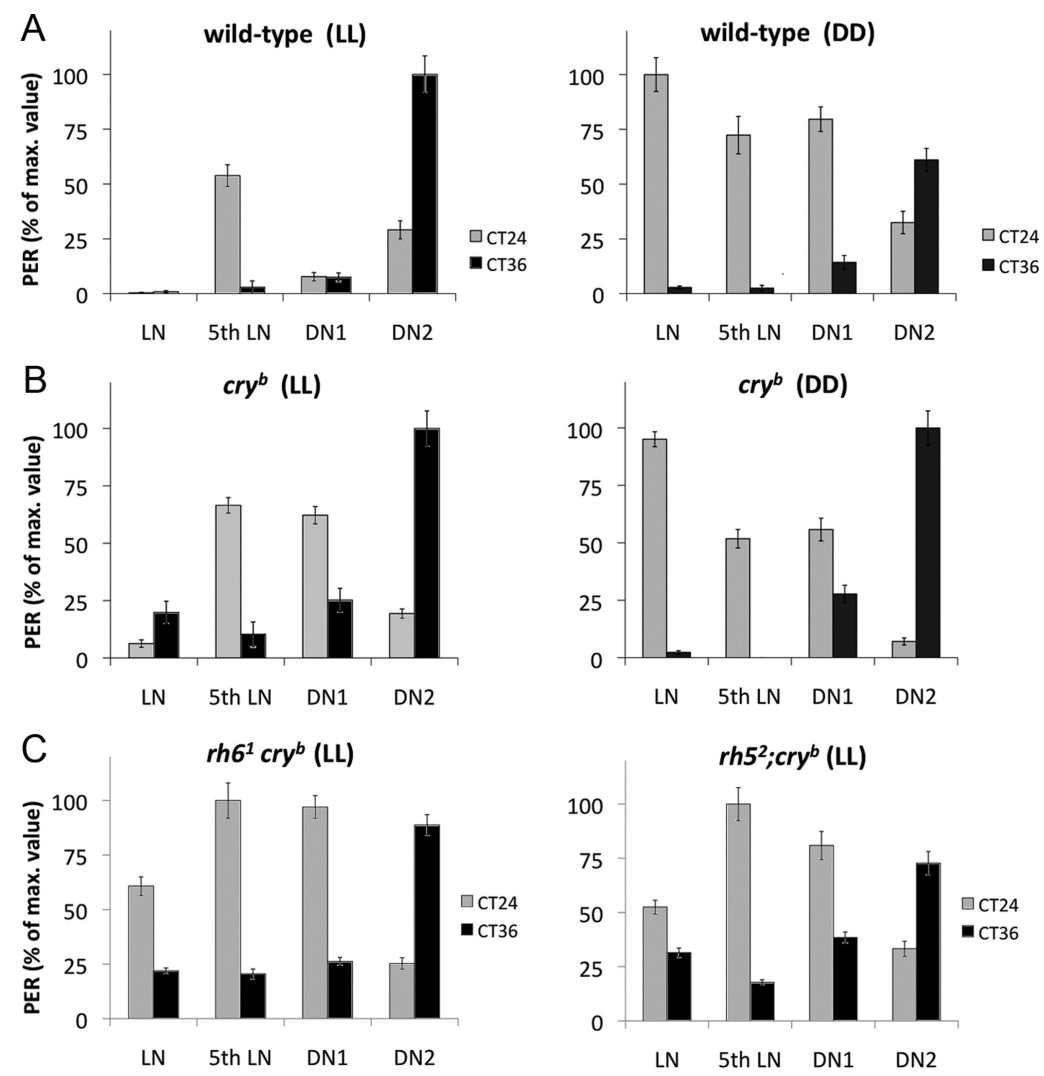

Figure 7. PER oscillations in constant light or constant darkness. Labeling and quantitation were performed as described in Figure 1. $\boldsymbol{A}, \boldsymbol{B}$, Wild-type $(\boldsymbol{A})$ and $\operatorname{cry}^{b}(\boldsymbol{B})$ third-instar larval brains were dissected during the second day in constant darkness or constant light following LD entrainment. In constant light, we consistently observed lack of PER expression for the wild-type PDF ${ }^{+}$LNs versus weak antiphase PER oscillations for the $\mathrm{cry}^{b} \mathrm{PDF}^{+}{ }^{+}$LNs. In contrast, the amplitude of PER oscillations in the DN2s was quite variable. $C, r h 6^{1} c r y^{b}$ and $r h 5^{2}$; $c r y{ }^{b}$ third-instar larval brains were dissected during the second day in constant light.
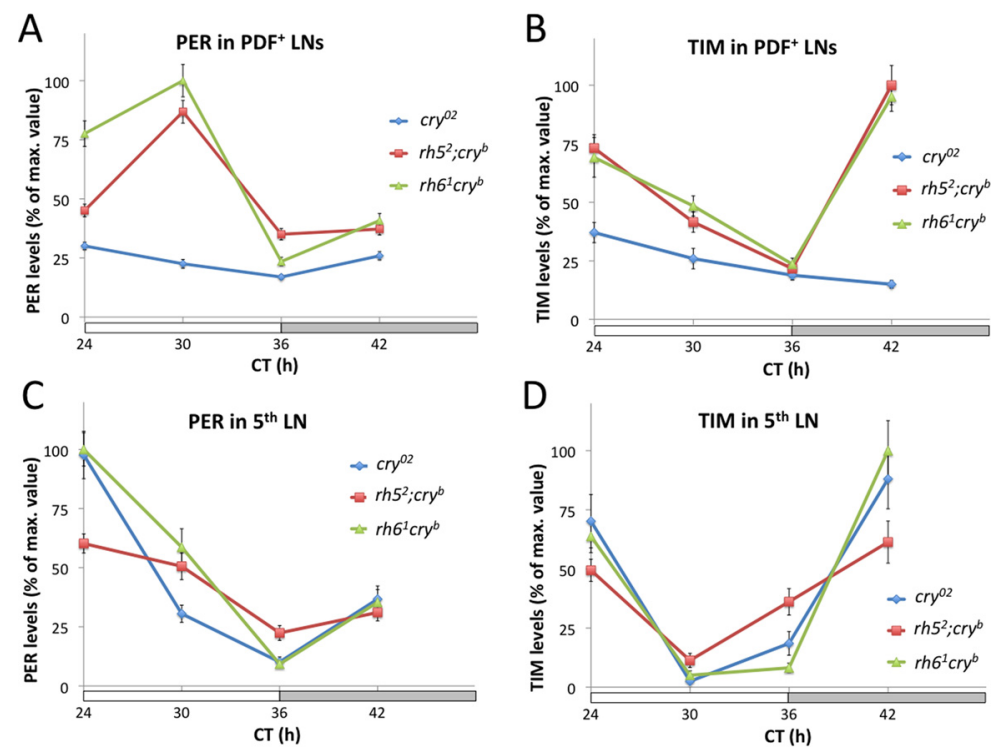

Figure 8. PER and TIM oscillations in constant light. Third-instar larval brains of the indicated genotypes were dissected during the second day in constant light, and simultaneously labeled with PDF, PER, and TIM antibodies. A, C, Quantitation of PER labeling in the four PDF ${ }^{+} \mathrm{LNs}_{(\boldsymbol{A})}$ and in the PDF ${ }^{-}$fifth $\mathrm{LN}(\boldsymbol{C}) . \boldsymbol{B}, \boldsymbol{D}$, Same for TIM labeling. White and light gray horizontal bars indicate the light and dark portions, respectively, of the preceding LD cycle. 

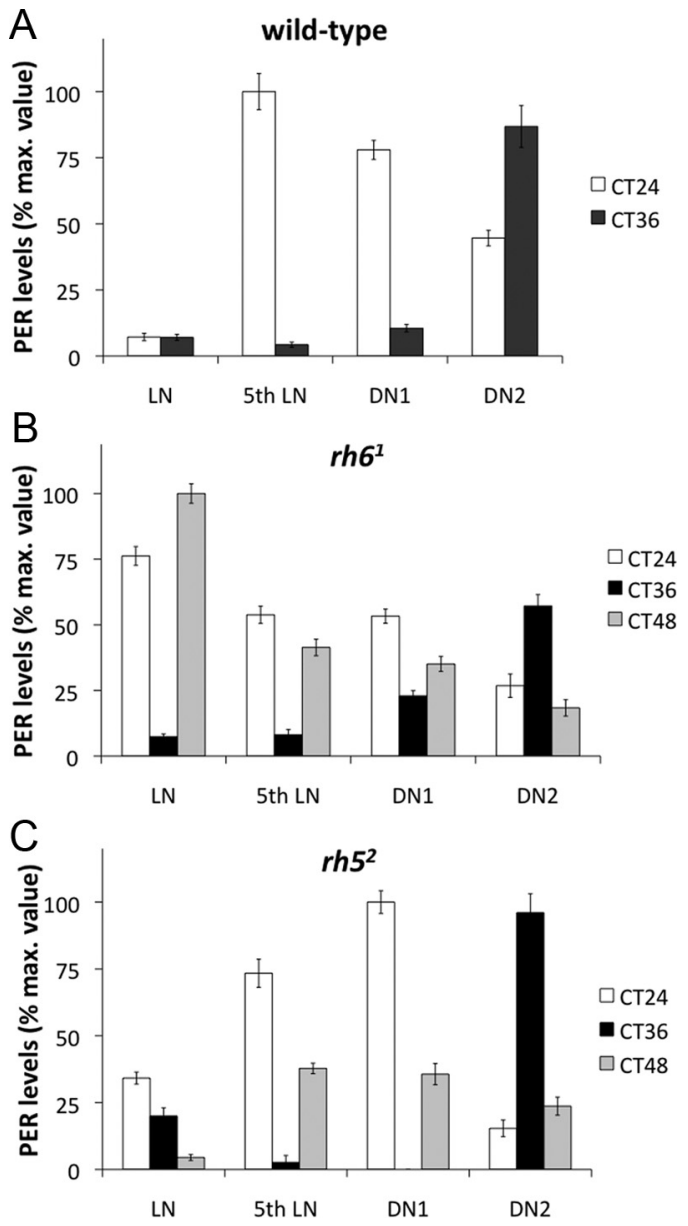

Figure 9. PER oscillations in constant red light. During the first day in constant red light, labeling and quantitation were performed as described in Figure 1 on brains of wild-type $(\boldsymbol{A}), r h 6^{1}(\boldsymbol{B})$, and $r h 5^{2}(\boldsymbol{C})$ larvae. In $\boldsymbol{A}$ and $\boldsymbol{B}$, the larvae also carried tim-gal4 and UAS-gfp as a reporter for clock neurons. Similar results were obtained in the absence of such reporting. An additional time point was dissected for $\boldsymbol{B}$ and $\boldsymbol{C}$ to better evaluate PER oscillations in the PDF ${ }^{+}$LNs.

trast, seem to respond normally even to a very short light pulse (Yang et al., 1998), suggesting that the visual system is dispensable for the response to light pulses in adults, but not larvae. The different sensitivity of the larval clock to visual system inputs could be related to the change in signaling pathways that occurs as the larval cholinergic visual system (Yasuyama et al., 1995) develops into the adult histaminergic visual system (Pollack and Hofbauer, 1991; Melzig et al., 1996). Moreover, contrary to the adult situation, the larval visual nerve may be light sensitive all along its length, down to its connection with the LNs, as judged from RH6 and NORPA expression. How visual system signaling ultimately affects the clock, whether in larvae or adults, remains to be discovered.

Both $\mathrm{RH}^{+}{ }^{+}$and $\mathrm{RH}^{+}{ }^{+} \mathrm{BO}$ photoreceptors contribute to the light responses of the larval brain clock that were tested, i.e., entrainment in LD and disruption of LN rythmicity in LL. Similarly, both photoreceptor types are equally able to suppress TIM levels in the LNs after a $2 \mathrm{~h}$ light exposure at the beginning of the night (Keene et al., 2011). In contrast, RH5 fibers alone specifically mediate acute larval responses to light (Hassan et al., 2005; Keene et al., 2011), while RH6 fibers alone are specifically required for the development of a serotonergic arborization that also contacts the LNs (Rodriguez Moncalvo and Campos, 2005). That RH6 activation strongly disrupts molecular oscillations in the LNs even in RR was, however, not anticipated. In the adult, RR does not affect molecular (A. Klarsfeld and F. Rouyer, unpublished results) or activity (Cusumano et al., 2009) rhythms.

Activation of RH6 above $600 \mathrm{~nm}$ is less than a few percent of peak activation at $\sim 510 \mathrm{~nm}$ (Salcedo et al., 1999). This suggests that the clock of the larval LN is extremely sensitive to red light, which may explain why no larval activity rhythm was recorded in a study that used video tracking in constant red light (Sawin et al., 1994). A strong sensitivity of larvae to the more penetrating, longer wavelengths of light may be related to their burrowing lifestyle.

\section{References}

Benito J, Zheng H, Hardin PE (2007) PDP1 $\varepsilon$ functions downstream of the circadian oscillator to mediate behavioral rhythms. J Neurosci 27:2539-2547.

Benito J, Houl JH, Roman GW, Hardin PE (2008) The blue-light photoreceptor CRYPTOCHROME is expressed in a subset of circadian oscillator neurons in the Drosophila CNS. J Biol Rhythms 23:296-307.

Bergmann A, Agapite J, McCall K, Steller H (1998) The Drosophila gene hid is a direct molecular target of Ras-dependent survival signaling. Cell 95:331-341.

Blanchardon E, Grima B, Klarsfeld A, Chélot E, Hardin PE, Préat T, Rouyer F (2001) Defining the role of Drosophila lateral neurons in the control of circadian activity and eclosion rhythms by targeted genetic ablation and PERIOD protein overexpression. Eur J Neurosci 13:871-888.

Cusumano P, Klarsfeld A, Chélot E, Picot M, Richier B, Rouyer F (2009) PDF-modulated visual inputs and Cryptochrome define diurnal behavior in Drosophila. Nat Neurosci 12:1427-1433.

Dolezelova E, Dolezel D, Hall JC (2007) Rhythm defects caused by newly engineered null mutations in Drosophila's cryptochrome gene. Genetics 177:329-345.

Dunlap JC, Loros JJ, DeCoursey PJ (2004) Chronobiology. Biological timekeeping. Sunderland, MA: Sinauer.

Emery P, Stanewsky R, Hall JC, Rosbash M (2000a) A unique circadianrhythm photoreceptor. Nature 404:456-457.

Emery P, Stanewsky R, Helfrich-Förster C, Emery-Le M, Hall JC, Rosbash M (2000b) Drosophila CRY is a deep brain circadian photoreceptor. Neuron 26:493-504.

Hanai S, Ishida N (2009) Entrainment of Drosophila circadian clock to green and yellow light by Rh1, Rh5, Rh6 and CRY. Neuroreport 20:755-758

Hanai S, Hamasaka Y, Ishida N (2008) Circadian entrainment to red light in Drosophila: requirement of Rhodopsin 1 and Rhodopsin 6. Neuroreport 19:1441-1444.

Hassan J, Iyengar B, Scantlebury N, Rodriguez Moncalvo V, Campos AR (2005) Photic input pathways that mediate the Drosophila larval response to light and circadian rhythmicity are developmentally related but functionally distinct. J Comp Neurol 481:266-275.

Helfrich-Förster C (1995) The period clock gene is expressed in central nervous system neurons which also produce a neuropeptide that reveals the projections of circadian pacemaker cells within the brain of Drosophila melanogaster. Proc Natl Acad Sci U S A 92:612-616.

Helfrich-Förster C, Winter C, Hofbauer A, Hall JC, Stanewsky R (2001) The circadian clock of fruit flies is blind after elimination of all known photoreceptors. Neuron 30:249-261.

Helfrich-Förster C, Edwards T, Yasuyama K, Wisotzki B, Schneuwly S, Stanewsky R, Meinertzhagen IA, Hofbauer A (2002) The extraretinal eyelet of Drosophila: development, ultrastructure and putative circadian function. J Neurosci 22:9255-9266.

Helfrich-Förster C, Shafer OT, Wülbeck C, Grieshaber E, Rieger D, Taghert P (2007a) Development and morphology of the clock-gene-expressing lateral neurons of Drosophila melanogaster. J Comp Neurol 500:47-70.

Helfrich-Förster C, Yoshii T, Wülbeck C, Grieshaber E, Rieger D, Bachleitner W, Cusamano P, Rouyer F (2007b) The lateral and dorsal neurons of Drosophila melanogaster: new insights about their morphology and function. Cold Spring Harb Symp Quant Biol 72:517-525.

Hofbauer A, Buchner E (1989) Does Drosophila have seven eyes? Z Naturforsch [C] 76:335-336.

Houl JH, Yu W, Dudek SM, Hardin PE (2006) Drosophila CLOCK is con- 
stitutively expressed in circadian oscillator and non-oscillator cells. J Biol Rhythms 21:93-103.

Houl JH, Ng F, Taylor P, Hardin PE (2008) CLOCK expression identifies developing circadian oscillator neurons in the brains of Drosophila embryos. BMC Neurosci 9:119.

Ivanchenko M, Stanewsky R, Giebultowicz JM (2001) Circadian photoreception in Drosophila: functions of cryptochrome in peripheral and central clocks. J Biol Rhythms 16:205-215.

Kaneko M (1998) Neural substrates of Drosophila rhythms revealed by mutants and molecular manipulations. Curr Opin Neurobiol 8:652-658.

Kaneko M, Hall JC (2000) Neuroanatomy of cells expressing clock genes in Drosophila: transgenic manipulation of the period and timeless genes to mark the perikarya of circadian pacemaker neurons and their projections. J Comp Neurol 422:66-94.

Kaneko M, Helfrich-Förster C, Hall JC (1997) Spatial and temporal expression of the period and timeless genes in the developing nervous system of Drosophila: newly identified pacemaker candidates and novel features of clock gene product cycling. J Neurosci 17:6745-6760.

Kaneko M, Hamblen MJ, Hall JC (2000) Involvement of the period gene in developmental time-memory: effect of the perShort mutation on phase shifts induced by light pulses delivered to Drosophila larvae. J Biol Rhythms 15:13-30.

Keene AC, Mazzoni EO, Zhen J, Younger MA, Yamaguchi S, Blau J, Desplan C, Sprecher SG (2011) Distinct visual pathways mediate Drosophila larval light avoidance and circadian clock entrainment. J Neurosci 31:6527-6534.

Klarsfeld A, Malpel S, Michard-Vanhée C, Picot M, Chélot E, Rouyer F (2004) Novel features of cryptochrome-mediated photoreception in the brain circadian clock of Drosophila. J Neurosci 24:1468-1477.

Lin Y, Stormo GD, Taghert PH (2004) The neuropeptide PDF coordinates pacemaker interactions in the Drosophila circadian system. J Neurosci 24:7951-7957.

Malpel S, Klarsfeld A, Rouyer F (2002) Larval optic nerve and adult extra-retinal photoreceptors sequentially associate with the clock neurons during Drosophila brain development. Development 129:1443-1453.

Malpel S, Klarsfeld A, Rouyer F (2004) Circadian synchronization and rhythmicity in larval photoperception-defective mutants of Drosophila. J Biol Rhythms 19:10-21.

Mazzoni EO, Desplan C, Blau J (2005) Circadian pacemaker neurons transmit and modulate visual information to control a rapid behavioral response. Neuron 45:293-300.

Melzig J, Buchner S, Wiebel F, Wolf R, Burg M, Pak WL, Buchner E (1996) Genetic depletion of histamine from the nervous system of Drosophila eliminates specific visual and mechanosensory behavior. J Comp Physiol A 179:763-773.

Misra S, Crosby MA, Mungall CJ, Matthews BB, Campbell KS, Hradecky P, Huang Y, Kaminker JS, Millburn GH, Prochnik SE, Smith CD, Tupy JL, Whitfied EJ, Bayraktaroglu L, Berman BP, Bettencourt BR, Celniker SE, de Grey AD, Drysdale RA, Harris NL, et al. (2002) Annotation of the Drosophila melanogaster euchromatic genome: a systematic review. Genome Biol 3:RESEARCH0083.
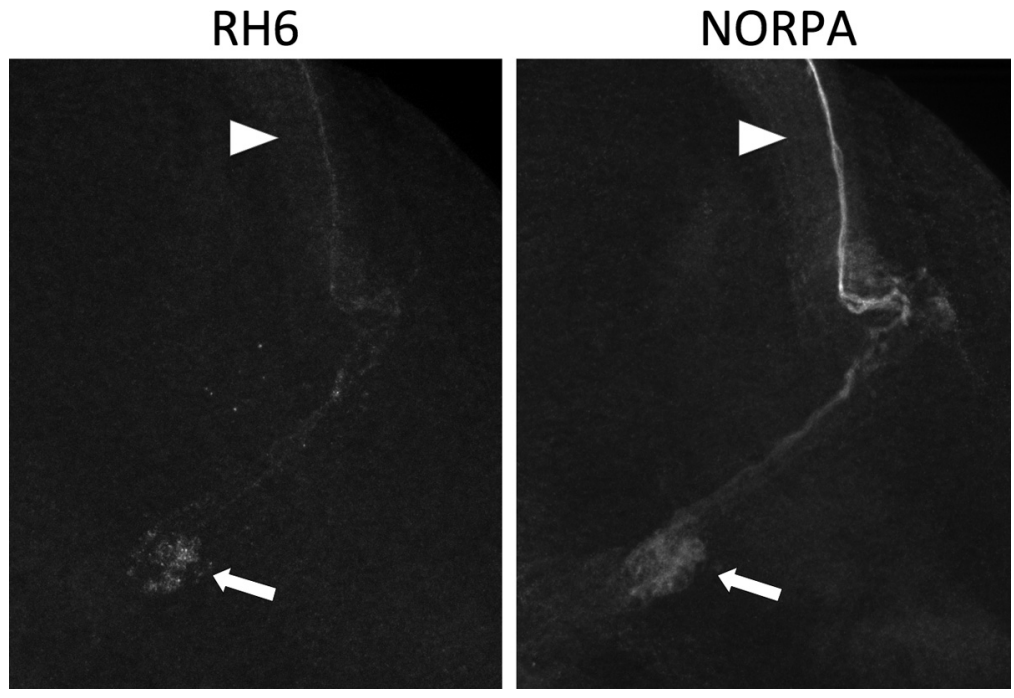

Figure 10. Components of the phototransduction cascade are present all along the larval visual nerve. Projection of confocal stacks of a third-instar larval brain double labeled with anti-RH6 and anti-NORPA antibodies. The arrow points at the terminal of Bolwig's nerve (BN). The relative intensities along the nerve suggest that RH6 is enriched in the terminal. The larger size of the NORPA-labeled versus the RH6-labeled terminal presumably indicates the presence of NORPA also in the RH5-expressing visual fibers, which make up one-third of the BN (Malpel et al., 2002; Sprecher et al., 2007). The arrowhead indicates the proximal part of the optic stalk, just before its connection to the brain.

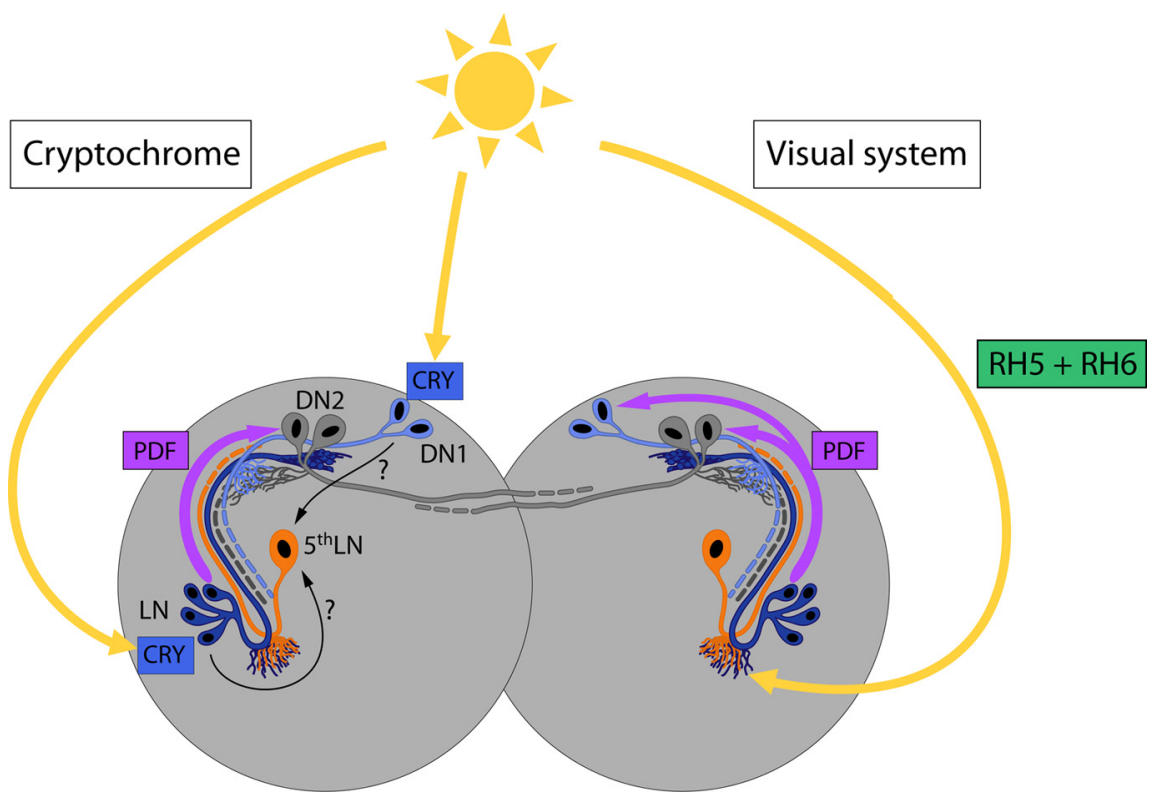

Figure 11. Schematic representation of light inputs into the brain clock of Drosophila larvae. For simplicity, the left hemisphere displays pathways active when only CRY is activated (visual system-ablated or $r h 5^{2}$; $r h 6^{7}$ mutant larvae), whereas the right hemisphere displays pathways active when only the visual system is activated (cry mutants). Each of the four neuronal groups has its own unique combination of light inputs, as indicated by different colors (Table 1). The $\mathrm{CRY}^{+}$neurons are in blue (dark blue for $\mathrm{PDF}^{+}$LNs; light blue for DN1s). In the absence of CRY, the DN1s require PDF signaling from the LNs to see light (arrow on right hemisphere). Thus, they have no direct connection to the visual system. The DN2s (gray) require PDF for entrainment both via the visual system and via CRY (arrow on both sides of the brain). The CRY ${ }^{-}$fifth LN (orange) can see light both via a direct connection to the visual system (like the other four LNs) and via a PDF-independent pathway, which enables entrainment even in the absence of the visual system. This pathway must originate from other $\mathrm{CRY}^{+}$cells, since CRY and the visual system are the only light inputs to the brain clock. The question marks thus indicate the possible involvement of a direct connection between the corresponding clock neurons.

Nitabach MN, Taghert PH (2008) Organization of the Drosophila circadian control circuit. Curr Biol 18:R84-R93.

Peng Y, Stoleru D, Levine JD, Hall JC, Rosbash M (2003) Drosophila freerunning rhythms require intercellular communication. PLoS Biol 1:e13.

Peschel N, Veleri S, Stanewsky R (2006) Veela defines a molecular link be- 
Table 1. Cryptochrome and the visual system define four combinations of light inputs to the clock neurons in the Drosophila larval brain

\begin{tabular}{lllll}
\hline & \multicolumn{3}{l}{ Neuronal group } & \\
\cline { 2 - 5 } Light inputs & $\mathrm{PDF}^{+} \mathrm{LN}$ & 5th $\mathrm{LN}$ & DN1 & DN2 \\
\hline CRY & $+^{a}$ & $-^{b}$ & + & via PDF \\
Visual system & + & + & via PDF & via PDF
\end{tabular}

$\overline{{ }^{a}+\text { indicates that these neurons can sense light via the corresponding input pathway without any intermediate }}$ neuronal connection.

${ }^{b}$ This neuron can also perceive light indirectly via $\mathrm{CRY}^{+}{ }^{+}$neurons, in a PDF-independent fashion.

tween Cryptochrome and Timeless in the light-input pathway to Drosophila’s circadian clock. Proc Natl Acad Sci U S A 103:17313-17318.

Picot M, Cusumano P, Klarsfeld A, Ueda R, Rouyer F (2007) Light activates output from evening neurons and inhibits output from morning neurons in the Drosophila circadian clock. PLoS Biol 5:e315.

Picot M, Klarsfeld A, Chélot E, Malpel S, Rouyer F (2009) A role for blind DN2 clock neurons in temperature entrainment of the Drosophila larval brain. J Neurosci 29:8312-8320.

Pollack I, Hofbauer A (1991) Histamine-like immunoreactivity in the visual system and brain of Drosophila melanogaster. Cell Tissue Res 266: 391-398.

Renn SC, Park JH, Rosbash M, Hall JC, Taghert PH (1999) A pdf neuropeptide gene mutation and ablation of PDF neurons each cause severe abnormalities of behavioral circadian rhythms in Drosophila. Cell 99:791-802.

Rieger D, Stanewsky R, Helfrich-Förster C (2003) Cryptochrome, compound eyes, $\mathrm{H}-\mathrm{B}$ eyelets and ocelli play different roles in the entrainment and masking pathway of the locomotor activity rhythm in the fruit fly Drosophila melanogaster. J Biol Rhythms 18:377-391.

Rieger D, Shafer OT, Tomioka K, Helfrich-Förster C (2006) Functional analysis of circadian pacemaker neurons in Drosophila melanogaster. J Neurosci 26:2531-2543.

Rodriguez Moncalvo VG, Campos AR (2005) Genetic dissection of trophic interactions in the larval optic neuropil of Drosophila melanogaster. Dev Biol 286:549-558.

Roenneberg T, Daan S, Merrow M (2003) The art of entrainment. J Biol Rhythms 18:183-194.

Salcedo E, Huber A, Henrich S, Chadwell LV, Chou WH, Paulsen R, Britt SG (1999) Blue- and green-absorbing visual pigments of Drosophila: ectopic expression and physiological characterization of the R8 photoreceptor cell-specific Rh5 and Rh6 rhodopsins. J Neurosci 19:10716-10726.

Sawin EP, Harris LR, Campos AR, Sokolowski MB (1994) Sensorimotor transformation from light reception to phototactic behavior in Drosophila larvae (diptera: Drosophilidae). J Insect Behav 7:553-567.

Shafer OT, Helfrich-Förster C, Renn SC, Taghert PH (2006) Reevaluation of Drosophila melanogaster's neuronal circadian pacemakers reveals new neuronal classes. J Comp Neurol 498:180-193.

Shen WL, Kwon Y, Adegbola AA, Luo J, Chess A, Montell C (2011) Function of rhodopsin in temperature discrimination in Drosophila. Science 331:1333-1336

Sprecher SG, Desplan C (2008) Switch of rhodopsin expression in terminally differentiated Drosophila sensory neurons. Nature 454:533-537.
Sprecher SG, Pichaud F, Desplan C (2007) Adult and larval photoreceptors use different mechanisms to specify the same Rhodopsin fates. Genes Dev 21:2182-2195.

Stanewsky R, Kaneko M, Emery P, Beretta B, Wager-Smith K, Kay SA, Rosbash M, Hall JC (1998) The cryb mutation identifies cryptochrome as a circadian photoreceptor in Drosophila. Cell 95:681-692.

Stoleru D, Peng Y, Agosto J, Rosbash M (2004) Coupled oscillators control morning and evening locomotor behavior of Drosophila. Nature 431:862-868.

Veleri S, Rieger D, Helfrich-Förster C, Stanewsky R (2007) HofbauerBuchner eyelet affects circadian photosensitivity and coordinates TIM and PER expression in Drosophila clock neurons. J Biol Rhythms 22:29-42.

Wernet MF, Mazzoni EO, Celik A, Duncan DM, Duncan I, Desplan C (2006) Stochastic spineless expression creates the retinal mosaic for colour vision. Nature 440:174-180.

Wülbeck C, Grieshaber E, Helfrich-Förster C (2008) Pigment-dispersing factor (PDF) has different effects on Drosophila's circadian clocks in the accessory medulla and in the dorsal brain. J Biol Rhythms 23:409-424.

Xiang Y, Yuan Q, Vogt N, Looger LL, Jan LY, Jan YN (2010) Lightavoidance-mediating photoreceptors tile the Drosophila larval body wall. Nature 468:921-926.

Yamaguchi S, Wolf R, Desplan C, Heisenberg M (2008) Motion vision is independent of color in Drosophila. Proc Natl Acad Sci USA, 105:4910-4915.

Yang Z, Emerson M, Su HS, Sehgal A (1998) Response of the timeless protein to light correlates with behavioral entrainment and suggests a nonvisual pathway for circadian photoreception. Neuron 21:215-223.

Yasuyama K, Meinertzhagen IA (1999) Extraretinal photoreceptors at the compound eye's posterior margin in Drosophila melanogaster. J Comp Neurol 412:193-202.

Yasuyama K, Kitamoto T, Salvaterra PM (1995) Localization of choline acetyltransferase-expressing neurons in the larval visual system of Drosophila melanogaster. Cell Tissue Res 282:193-202.

Yasuyama K, Okada Y, Hamanaka Y, Shiga S (2006) Synaptic connections between eyelet photoreceptors and pigment dispersing factor-immunoreactive neurons of the blowfly Protophormia terraenovae. J Comp Neurol 494:331-344.

Yoshii T, Todo T, Wülbeck C, Stanewsky R, Helfrich-Förster C (2008) Cryptochrome is present in the compound eye and a subset of Drosophila's clock neurons. J Comp Neurol 508:952-966.

Yoshii T, Wülbeck C, Sehadova H, Veleri S, Bichler D, Stanewsky R, HelfrichFörster C (2009) The neuropeptide pigment-dispersing factor adjusts period and phase of Drosophila's clock. J Neurosci 29:2597-2610.

Zhang L, Chung BY, Lear BC, Kilman VL, Liu Y, Mahesh G, Meissner RA, Hardin PE, Allada R (2010) DN1(p) circadian neurons coordinate acute light and PDF inputs to produce robust daily behavior in Drosophila. Curr Biol 20:591-599.

Zhou L, Schnitzler A, Agapite J, Schwartz LM, Steller H, Nambu JR (1997) Cooperative functions of the reaper and head involution defective genes in the programmed cell death of Drosophila central nervous system midline cells. Proc Natl Acad Sci U S A, 94:5131-5136. 